\title{
Predicting sandy soil moisture content with hyperspectral imaging
}

\author{
Qi Haijun ${ }^{1}$, Jin Xiu ${ }^{1}$, Zhao Liu ${ }^{1}$, DEDO Irene Maxime ${ }^{1,2}$, Li Shaowen ${ }^{1 *}$ \\ (1. School of Information and Computer, Anhui Agricultural University, Hefei 230036, China; \\ 2. University of Abomey-Calavi, Cotonou, Benin)
}

\begin{abstract}
In this study, a rapid and non-invasive technology for predicting soil moisture content (SMC) was presented based on hyperspectral imaging (HSI). Firstly, a set of HSI system was developed to collect both spectral (400-1000 nm) and spatial $(1620 \times 841$ pixels) information from sandy soil samples with variable SMC levels in the laboratory. Principal component analysis (PCA) transformation, $K$-means clustering, and several other image processing methods were performed to obtain a region of interest (ROI) of soil sample from the original HSI data. Then, 256 optimal spectral wavelengths were selected from the average reflectance of the ROI, and 28 textural features were extracted using a gray-level co-occurrence matrix (GLCM). Data dimensionality reduction was conducted on both the spectral information and textural information by using a partial least square algorithm. Six latent variables (LVs) extracted from the spectral information, four LVs extracted from the textural information and fused data were used to build regression models with a three-layer BPNN, respectively. The results showed that all of the three calibration models achieved high prediction accuracy, particularly when using spectral information with $R_{C}^{2}=0.9532$ and $R M S E C=0.0086$. However, validation models demonstrate that predicting SMC using fused data is more effective than using spectral reflectance and textural features separately, with a $R_{P}^{2}=0.9350$ and $R M S E P=0.0141$, thus proving that the HSI technique is capable of detecting SMC.
\end{abstract}

Keywords: hyperspectral imaging, soil moisture content, image processing, prediction model, fused data, BPNN, regression DOI: $10.25165 / j$.jjabe.20171006.2614

Citation: Qi H J, Jin X, Zhao L, DEDO I M, Li S W. Predicting sandy soil moisture content with hyperspectral imaging. Int J Agric \& Biol Eng, 2017; 10(6): 175-183.

\section{Introduction}

Soil moisture content (SMC) is an important parameter in crop growth and produces a significant variation in soil spectral reflectance. Scientists both at home and abroad have made great efforts to measure SMC by using spectroscopic methods. Gaussian models ${ }^{[1]}$, semi-empirical models ${ }^{[2]}$, and multivariate

\section{Received date: 2016-06-01 Accepted date: 2017-06-14}

Biographies: Qi Haijun, PhD candidate, research interest: soil spectroscopy, Email: haijun.qi0418@qq.com; Jin Xiu, PhD candidate, Lecturer, research interest: agricultural informatics, Email: jinxiu123@ahau.edu.cn; Zhao Liu, Master student, research interest: soil spectroscopy, Email: liulianjushi@126.com; DEDO Irene Maxime, $\mathrm{PhD}$ candidate, research interest: agricultural informatics, Email: maximededo@gmail.com.

*Corresponding author: Li Shaowen, $\mathrm{PhD}$, Professor, research interests: agricultural information detection and processing. Anhui Agricultural University, No.130 West Changjiang Road, Hefei 230036, China. Email: shwli@ahau.edu.cn. linear prediction models ${ }^{[3]}$ have been established in the optical domain of 1.2-2.5 $\mu \mathrm{m}, 0.4-14 \mu \mathrm{m}$ and 1390$1623 \mathrm{~nm}$, respectively, to estimate SMC by laboratory spectra. Recent studies $^{[4]}$ have demonstrated that hyperspectral analysis as an effective approach on predicting SMC and the transformations of spectra have the ability to improve the accuracy of the prediction model. The determination coefficient $\left(R^{2}\right)$ was 0.931 when using the spectral reflectance logarithm of the first derivative differential to estimate the SMC in black soil by using stepwise multiple linear regression ${ }^{[5]}$. The wavelet transform method can be used to improve the $R^{2}$ by dramatically reducing the dimensionality of hyperspectral data ${ }^{[6]}$. The $R^{2}$ was also improved when moving from controlled laboratory conditions to field conditions using clay content information ${ }^{[7]}$.

Hyperspectral imaging (HSI) integrates the main features of spectroscopic and imaging techniques, which can enhance the ability to detect minor or more complex 
features or characteristics of an object both spatially and spectrally ${ }^{[8]}$. HSI has been identified as a non-invasive technique for the rapid and effective prediction of basic components and nutritious/toxic elements in soil under laboratory condition, such as organic carbon ${ }^{[9]}$, organic matter and carbon fraction ${ }^{[10,11]}$, biochar particles and biochar concentration $^{[12]}$, chloride salt concentration ${ }^{[13]}$, clay content ${ }^{[14]}$ and total nitrogen $(\mathrm{TN})$ content for entire soil profiles ${ }^{[15]}$.

In this study, a dataset of hyperspectral images (400 $1000 \mathrm{~nm}$ ) of soil samples with variable SMC levels was collected by an HSI system in the laboratory, which was used to predict SMC with a three-layer BPNN regression model with spectral information, textural information and fused data.

\section{Materials and methods}

\subsection{Soil samples}

Yellow-brown sandy soil from a rapeseed field located in Hefei, China, was selected for this study. Three quadrates of $50 \mathrm{~cm} \times 50 \mathrm{~cm}$ were selected as sampling units following a random survey methodology. In each quadrate, approximately $5 \mathrm{~kg}$ of soil was excavated from a depth of 0-20 $\mathrm{mm}$ after a rainfall and collected in a sealed plastic bag that was taken to the laboratory. After leaves, roots and gravel were removed, a $2 \mathrm{~mm}$ sieve was used to separate fine soil from each of three soils, and the fractions passing through the sieve were used for measurement and analysis.

Progressive drying ${ }^{[2]}$ and constant water injections ${ }^{[5]}$ have been used to obtain successive SMC levels. Both techniques would create a difference in SMC between the surface and inside of a soil sample. Therefore, progressive natural evaporation was selected in this study. The soils collected were spread evenly on three $1 \mathrm{~m}^{2}$ desks, and labeled with 1, 2 or 3, based on the sample site. The experiment was conducted in the laboratory under normal indoor conditions with a temperature of $24^{\circ} \mathrm{C}$ and no wind. Each evaporation cycle endured $1 \mathrm{~h}$. As each step was completed, two samples of the soil on the desk were quickly removed into a dry petri dish (diameter of $90 \mathrm{~mm}$ and thickness of $15 \mathrm{~mm}$ ) or a weighed aluminum box (diameter of $46 \mathrm{~mm}$ and thickness of $25 \mathrm{~mm}$ ). The dishes were leveled at the rim with a spatula and then used for HSI data acquisition. The boxes were weighed both immediately and again after drying to obtain a SMC value. The whole period of evaporation lasted $11 \mathrm{~h}$, during which 36 soil samples were made and tested in total.

\subsection{SMC measurement}

The over-drying method ${ }^{[16]}$ is used to determine SMC value. All soil samples were dried in the laboratory oven (Jinghong, Shanghai, China), at a temperature of $105^{\circ} \mathrm{C}$ for $12 \mathrm{~h}$. Aluminum boxes and soil samples were weighed by a scale with an accuracy of $0.0001 \mathrm{~g}$. The SMC value (in \%) was calculated as:

$$
S M C=\frac{m_{1}-m_{2}}{m_{1}-m_{0}}
$$

where, $m_{1}$ is the weight of the wet sample, $\mathrm{g} ; m_{2}$ is the weight of the completely dried sample, $\mathrm{g} ; m_{0}$ is the weight of the completely dried aluminum box, $g$.

\subsection{HSI system and data acquisition}

Spectra and images of each spectral channel from the soil samples were acquired simultaneously using an indoor HSI system (Figure 1) developed by our research group. The system consists of five major components: a push-broom-type hyperspectral imager (OKSI, Torrance, CA, USA), a rotating stage with pan/tilt head for scanning, a light source comprised of two $50 \mathrm{~W}$ halogen lamps, a Dell Precision Workstation with HyperVision data collection software, and a set of height-adjustable mounting brackets.

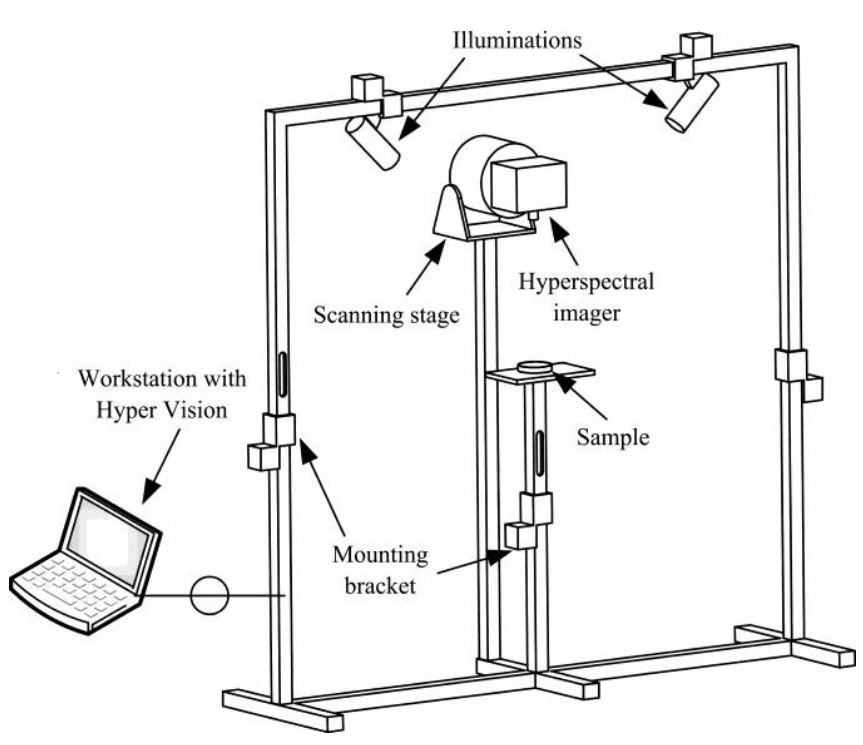

Figure 1 HSI system 
The HSI experiment was conducted in a darkroom to avoid the interference of outside light. Each soil sample was placed in a fixed location, and a piece of black light-absorbed cloth was placed beneath the target object as background. The output of this system was 'image cubes' of soil samples, which consisted of a two-dimensional spatial image $(1620 \times 841$ pixels $)$ with spectral data (400-1000 nm, 339 spectral channels) at each pixel. Several critical parameters of the system and experiment were set as shown in Table 1, based on repeated tests and comparisons.

Table 1 Critical system and experiment parameters

\begin{tabular}{lc}
\multicolumn{1}{c}{ Parameters } & Values \\
\hline Zenith angle $/\left(^{\circ}\right)$ & 45 \\
Distance of imager and sample/mm & 300 \\
Distance of illumination and sample/mm & 400 \\
Scan angle $/\left(^{\circ}\right)$ & 10 \\
Scan speed $/\left(^{\circ}\right) \cdot \mathrm{s}^{-1}$ & 0.519 \\
Exposure time $/ \mathrm{ms}$ & 10 \\
Pixel size/um & 8 \\
Focal length/mm & 17 \\
\hline
\end{tabular}

\subsection{Data processing and regression analysis}

\subsubsection{Region of interest (ROI) selection}

The original hyperspectral image is composed of the soil sample, petri dish, and black background. To efficiently and totally isolate the soil reflective part of the hyperspectral image as the ROI, principal component analysis (PCA), image interactive stretching, $K$-means clustering algorithm, median filtering and morphological operations ${ }^{[17]}$ were performed to generate the ROI for each soil sample (Figure 3).

\subsubsection{Calculation of reflectance}

The reflectance of soil samples $R_{I}$ of each wavelength in each pixel was determined based on the standard reflectance of a white panel (Labsphere, North Sutton, NH, USA).

The uncalibrated Digital Number (DN) value of the soil sample $D N_{I}$ and the white panel $D N_{W}$ are both composed of the signal of reflection brightness, dark current and noise, as follows:

$$
\begin{gathered}
L \cdot R_{I} \cdot \eta+D N_{B}=D N_{I} \\
L \cdot R_{W} \cdot \eta+D N_{B}=D N_{W}
\end{gathered}
$$

where, $L$ is the luminosity of incident light, cd; $\eta$ is the conversion rate of the hyperspectral imager; $R_{W}$ is the reflectance of the white panel; $D N_{B}$ is the DN used as a substitute for dark current and noise when the camera shutter is closed.

As can be inferred from Equations (2) and (3), the reflectance $R_{I}$ can be calculated as ${ }^{[18]}$.

$$
R_{I}=\frac{D N_{I}-D N_{B}}{D N_{W}-D N_{B}} \times R_{W}
$$

\subsubsection{Feature extraction}

The reflectance of ROI was averaged as a research basis for predicting the $\mathrm{SMC}^{[19,20]}$. The wavelengths at both ends have low signal to noise (SNR), which were removed to reduce the effect of predicting accuracy. The reflectance of 256 spectral channels (486.14$942.59 \mathrm{~nm})$ was selected and smoothed using the Savitzky-Golay Filtering algorithm ${ }^{[21]}$ to serve as spectral information for analysis.

PCA was performed again for the masked ROI from $486.14 \mathrm{~nm}$ to $942.59 \mathrm{~nm}$, and PC1 and PC2 (with over $99 \%$ of the cumulative percentage of variance) were selected to extract image textural information using a Gray-level co-occurrence matrix (GLCM) with a relative distance of $1^{[22]}$. The normalized mean and standard deviation $(\sigma)$ of dissimilarity, entropy, contrast, homogeneity, correlation, energy and peak in the relative orientations of $0^{\circ}, 45^{\circ}, 90^{\circ}$ and $135^{\circ}$ were used to describe the textural information.

To reduce the dimensions of original, experimentally derived dataset and improve the robustness of BPNN calibration ${ }^{[23]}$, the partial least square (PLS) algorithm was applied to the data to extract more sensitive features from the spectral and textural information ${ }^{[24,25]}$. The number of latent variables (LVs) was determined by the percentage of variance explained in the response variables (SMC) and the mean squared errors (MSE) with 10-fold cross validation ${ }^{[25]}$. The extracted LVs, as described above, served as the input features of the BPNN model.

\subsubsection{Regression with BPNN}

A three-layer feed-forward backpropagation neural network (BPNN) ${ }^{[26]}$ was used to establish the regression relationship between SMC and the LVs of the spectral information (Figure 2). 


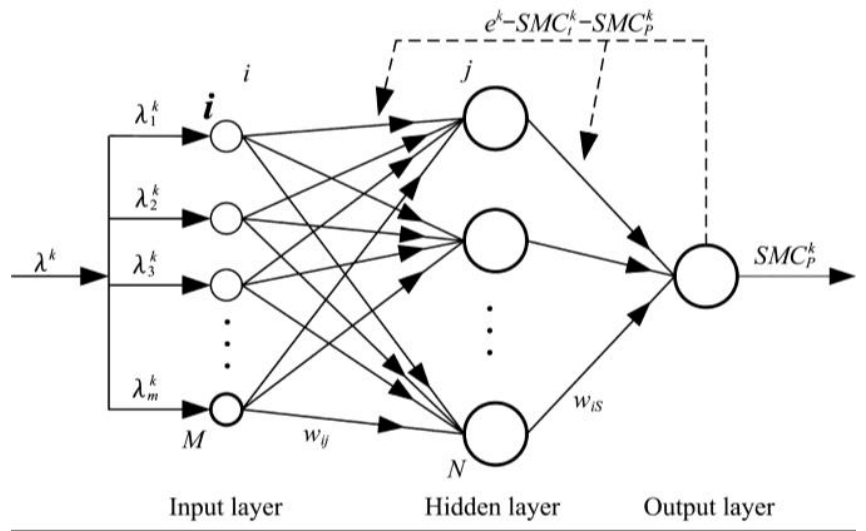

Figure 2 BPNN structure

$\lambda^{k}$, including $M$ LVs of the $k^{\text {th }}$ sample, was used as the input nodes. The output of network $S M C_{P}^{k}$ was the predicted value of SMC.

The outputs of the hidden and output layers are calculated as:

$$
\begin{gathered}
O_{j}^{k}=g_{1}\left(\sum_{i=1}^{M} w_{i j} \lambda_{i}^{k}\right) \\
S M C_{P}^{k}=g_{2}\left(\sum_{j=1}^{N} w_{j S} O_{j}^{k}\right)
\end{gathered}
$$

where, $g_{1}(\cdot)$ is the transfer function of the hidden layer; $w_{i j}$ is the weight connecting the input layer node $i$ and the hidden layer node $j ; g_{2}(\cdot)$ is the transfer function of the output layer; and $w_{j S}$ is the weight connecting the hidden layer node $j$ and the output layer node.

The mean squared error of $K$ training samples was calculated as:

$$
e=\frac{1}{2} \sum_{k=1}^{K}\left(S M C_{t}^{k}-S M C_{P}^{k}\right)^{2}
$$

where, $S M C_{t}^{k}$ is the target output, the actual SMC value of the $k^{\text {th }}$ sample.

If the mean squared error is higher than the set value, then the backpropagation would execute to correct the connection weights using the following formula until the error is smaller than that:

$$
\begin{gathered}
w_{i j}=w_{i j}+\left(-\eta \frac{\partial e}{\partial w_{i j}}\right) \\
w_{j S}=w_{j S}+\left(-\eta \frac{\partial e}{\partial w_{j S}}\right)
\end{gathered}
$$

where, $\eta$ is the learning rate.

The BPNN regression models were also built based on the LVs from the textural information and fused data. The stability and capacity of these prediction models were validated and compared with the determination coefficients $\left(R^{2}\right)$ and root mean square errors (RMSE).

\section{Results and analysis}

\subsection{SMC levels}

The SMC values of the 36 samples obtained from the three evaporating desks is shown in Table 2, which shows the gradient characteristics. The samples on the first and second desks were used as the calibration set to develop the regression model, and the rest of the samples formed the validation set.

Table 2 SMC values of 36 samples

\begin{tabular}{cccc}
\hline \multirow{2}{*}{$\begin{array}{c}\text { Evaporating } \\
\text { period/h }\end{array}$} & 1 & SMC/\% \\
\cline { 2 - 4 } 0 & 17.58 & 17.67 & 3 \\
\hline 1 & 16.98 & 17.39 & 18.03 \\
2 & 16.51 & 16.46 & 16.38 \\
3 & 16.17 & 15.44 & 12.75 \\
4 & 15.48 & 13.32 & 11.38 \\
5 & 15.05 & 12.51 & 10.03 \\
6 & 13.88 & 11.67 & 8.77 \\
7 & 12.20 & 11.45 & 7.76 \\
8 & 11.47 & 9.19 & 6.02 \\
9 & 10.46 & 7.37 & 4.26 \\
10 & 9.08 & 6.05 & 2.08 \\
11 & 8.13 & 2.63 & 0.56 \\
\hline
\end{tabular}

\subsection{Image processing and analysis}

\subsubsection{ROI selection}

The process and results of ROI selection are shown in Figure 3. As seen from the PCA results, the common reflective region of soil and Petri dish is represented by PC1, and the obvious feature of the Petri dish is found in PC4. Image contrasts were enhanced by interactive stretching, and some of the soil was reinforced in the stretched PC4. A new image was obtained by covering the stretched PC4 with extracted coordinates. This was used to subtract the stretched PC4 to segment the border of the soil. After image processing steps using different methods, the ROI of the soil reflective region was generated.

\subsubsection{Textural correlation analysis}

Twenty-eight textural features were extracted from PC1 and PC2 using the masked ROI, and the correlation coefficients between the textural features and SMC are shown in Table 3. The correlation between the textural features and SMC is quite clear. Notably, the $\sigma$ of energy has a strong negative correlation, with a 
coefficient of -0.8945 . However, poor correlation still exists among several textural features. Thus, seven textural features (numbers in Table 3 in italic font) were removed for an absolute value of coefficient less than 0.5 , and the other 21 textural features were used to predict $\mathrm{SMC}$, with a sequential input from the mean of PC1 and $\mathrm{PC} 2$ to the $\sigma$ of PC1 and PC2.

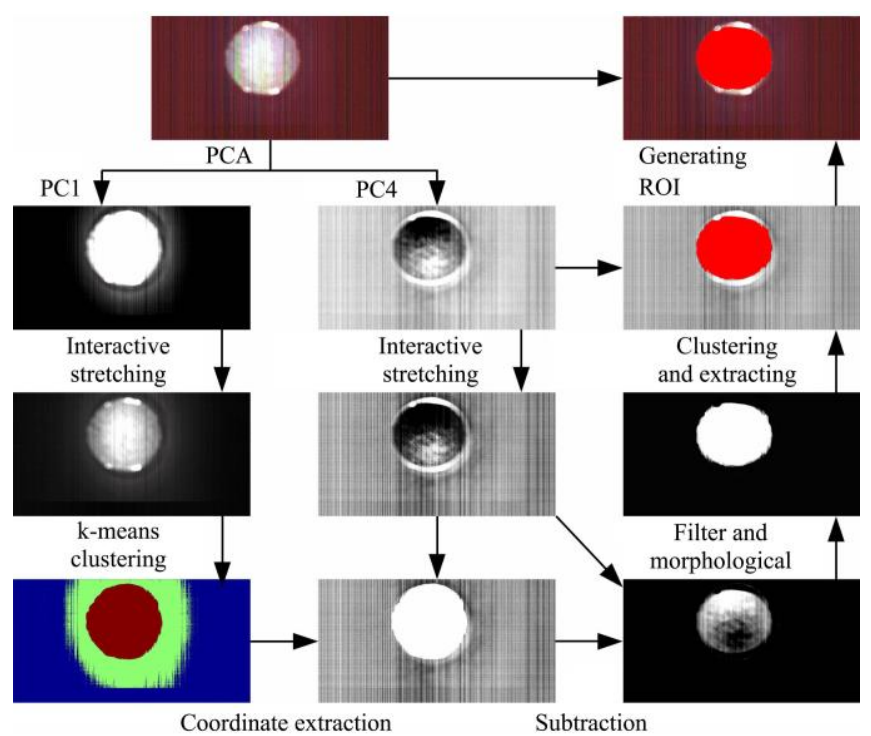

Figure 3 Process of ROI selection

Table 3 Correlation coefficient between texture and SMC

\begin{tabular}{|c|c|c|c|c|}
\hline \multirow{3}{*}{ texture feature } & \multicolumn{4}{|c|}{ correlation coefficient } \\
\hline & \multicolumn{2}{|c|}{$\mathrm{PC} 1$} & \multicolumn{2}{|c|}{$\mathrm{PC} 2$} \\
\hline & mean & $\sigma$ & mean & $\sigma$ \\
\hline dissimilarity & -0.6244 & -0.7781 & 0.6456 & 0.6606 \\
\hline entropy & -0.6102 & -0.6880 & 0.6464 & 0.6512 \\
\hline contrast & -0.4702 & -0.8737 & 0.3818 & 0.5634 \\
\hline homogeneity & 0.6312 & -0.8428 & -0.6472 & 0.6632 \\
\hline correlation & 0.5985 & -0.6800 & -0.8876 & 0.8783 \\
\hline energy & 0.3229 & -0.8945 & -0.0752 & 0.2120 \\
\hline peak & -0.0676 & $-\mathbf{0 . 5 8 9 7}$ & 0.2137 & 0.7554 \\
\hline
\end{tabular}

\subsection{Spectral characteristics}

Twelve samples from the first desk and 2 samples from other desks with different SMC values were selected to analyze spectral characteristics. The calibrated and smoothed reflectance values of samples with high and low SMC levels are shown in Figures $4 \mathrm{a}$ and $4 \mathrm{~b}$. The mean and standard deviation (STD) of reflectance are also presented. As seen, the reflectance of the soil samples generally increases with an increase in wavelength, ranging from $486.14 \mathrm{~nm}$ to $942.59 \mathrm{~nm}$, except for a slight upward trend around $820 \mathrm{~nm}$. The reflectance variation of different samples also increases as the wavelength increases; thus, the STD appears as an increasing trend. Both the mean and the STD show similar trends at high and low levels, but the variation is much obvious at low levels.

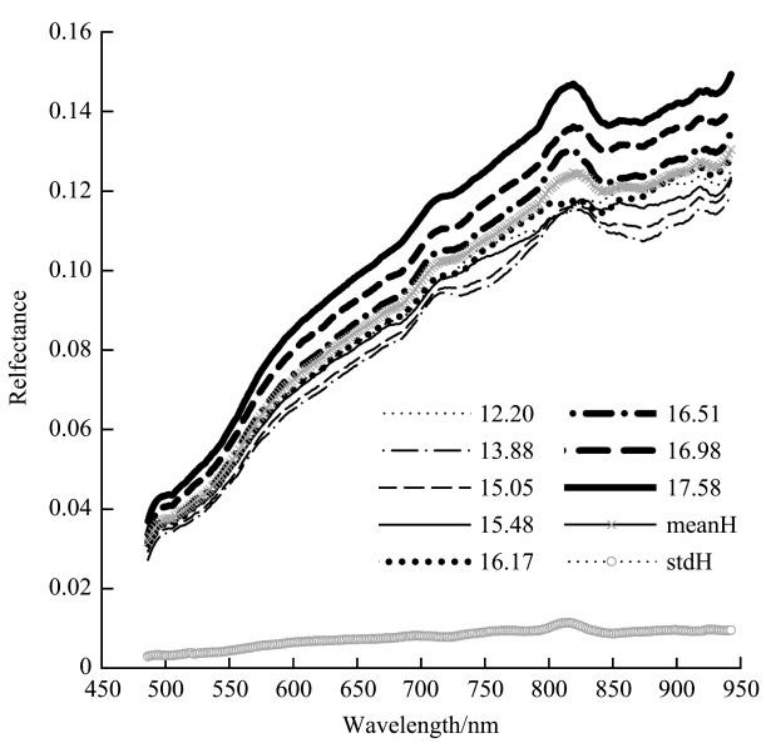

a. Reflectance of 8 samples at high SMC (\%) level

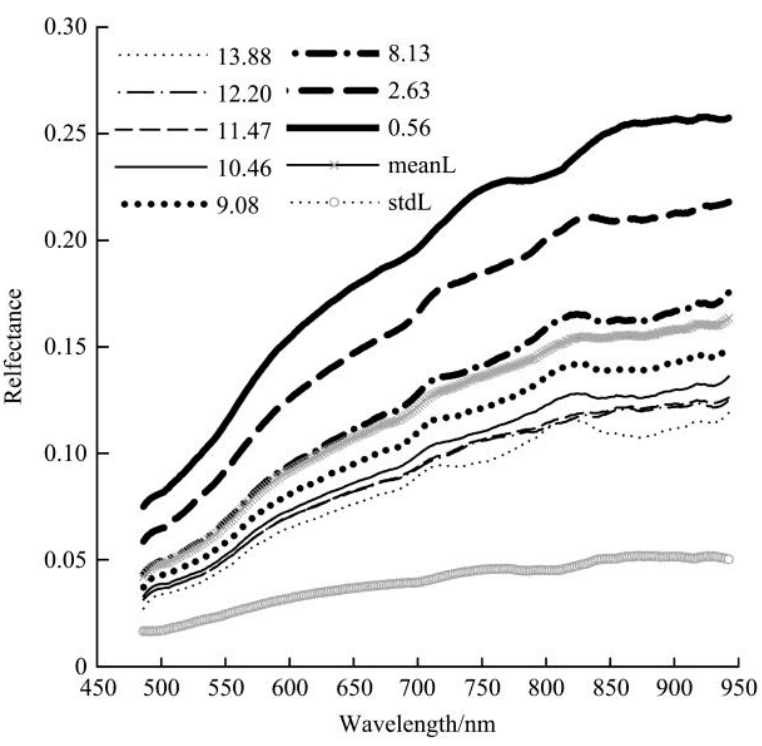

b. Reflectance of 8 samples at low SMC (\%) level

Figure 4 Spectral characteristics of 14 samples

According to literature [3-5], when SMC is lower than the field capacity, the reflectance of soil decreases as SMC increases, whereas it will increase when SMC is higher. As shown in Figures $4 \mathrm{a}$ and $4 \mathrm{~b}$, the reflectance decreases gradually with a longer evaporation duration in the first six hours and then becomes an increasing for the rest of the cycle. It can be concluded that the field capacity of yellow-brown sandy soil is approximately $13.88 \%$.

\subsection{Data dimensionality reduction}

The problems of data redundancy and collinearity should be considered because the overall reflectance shows similar changing characteristics, as shown in 
Figure 4. Therefore, it is important to reduce the dimensions of the spectral information.

PLS was performed on the training set with both spectral and textural information to extract the optimal number of LVs. The cumulative explained variance and MSE versus the number of LVs used in within spectral and textural information are presented in Figures 5a and $5 b$, respectively. The optimal number of LVs was selected based on the minimum MSEcv using 10-fold cross-validation. Six LVs extracted from the spectral information explained $98.73 \%$ of observed variance, and $4 \mathrm{LVs}$ extracted from the textural information explained $67.65 \%$ of observed variance; these LVs were selected to predict SMC. Using this approach, the number of input features for the BPNN regression model can be greatly reduced.

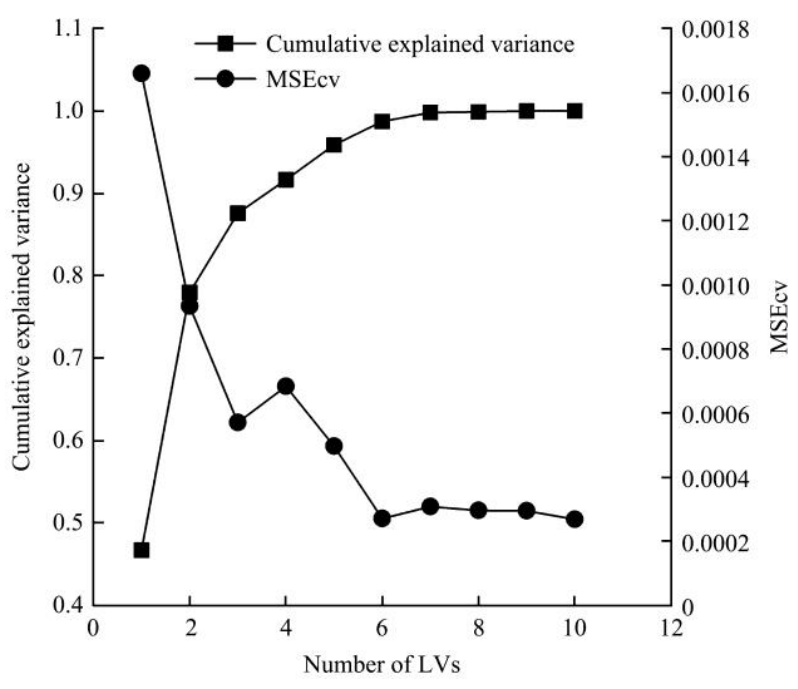

a. Spectral information

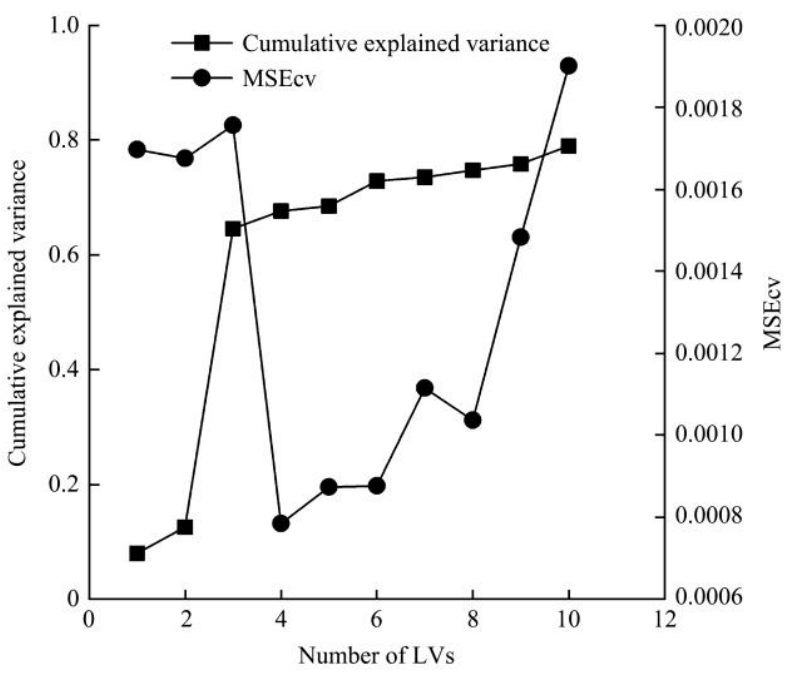

b. Textural information

Figure 5 Number of LVs used in PLS versus cross-validated cumulative explained variance and MSEcv

\subsection{Regression analysis}

\subsubsection{Regression with spectral information}

Six LVs extracted from the spectral information were applied as the input nodes for the BPNN. Tansig and logsig were selected as the transfer functions for the hidden and output layers, respectively. In addition, a backprop network training function was set to the Levenberg-Marquard algorithm (trainlm). The number of hidden layer nodes was set at 15 , and the learning rate, momentum factor, largest training number and expected model prediction error were set at 0.1, 0.6, 2000, and 1e-4, respectively. Thus, a three-layer BPNN model was established, which resulted in a best $R_{C}^{2}$ value of 0.9532 for the calibration set and a $R_{P}^{2}$ value of 0.9126 for the validation set (Figure 6a).

\subsubsection{Regression with textural features}

The same modeling approach was used to establish the BPNN regression relationship using the $4 \mathrm{LVs}$ extracted from the textural information as the input nodes, which generated a best $R_{C}^{2}$ value of 0.9386 (Figure 6b). However, the determination coefficient of the validation set resulted in 0.7196 due to only a moderate correlation (0.5-0.8) between SMC and several textural features (Table 3). According to Figure 5, LVs from the textural information can explain less variance than the spectral information. This is consistent with the predicted results.

\subsubsection{Regression with fused data}

Ten variables, fused with 6 LVs extracted from spectral information and $4 \mathrm{LVs}$ extracted from textural information, were applied as the input nodes to the established BPNN regression model, which resulted in a best $R_{C}^{2}$ value of 0.9492 (Figure 6c). The combination of these data expanded the amount of information and improved the collinearity and redundancy. As a result, the calibration model using the fused data generated a smaller $R_{C}^{2}$ than did the use of spectral information alone. However, benefitting from a wealth of information, the validation models illustrate that using fused data to predict SMC achieved a $R_{P}^{2}$ of 0.9350 , which is better than using spectral information.

\subsection{Interpretation of the prediction ability}

The loading weights can be considered as indicators 
for the 'correlations' between the property of interest and the input features ${ }^{[27]}$. As can be inferred from Figure 5, the first three LVs are the most important for predicting
SMC. Therefore, the loading weights of the first three LVs for the spectral and textural information were centered on zero ${ }^{[28]}$ and are presented in Figure 7.
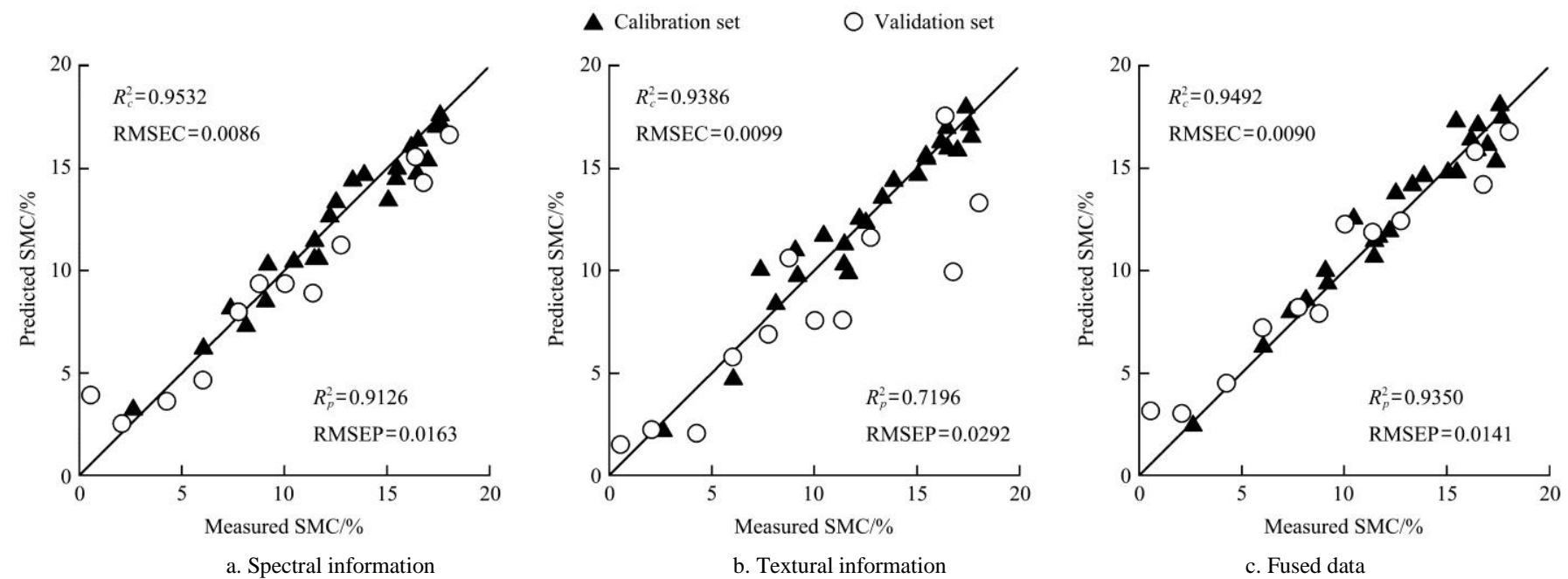

Figure 6 Measured versus predicted values of SMC using spectral information, textural information and fused data
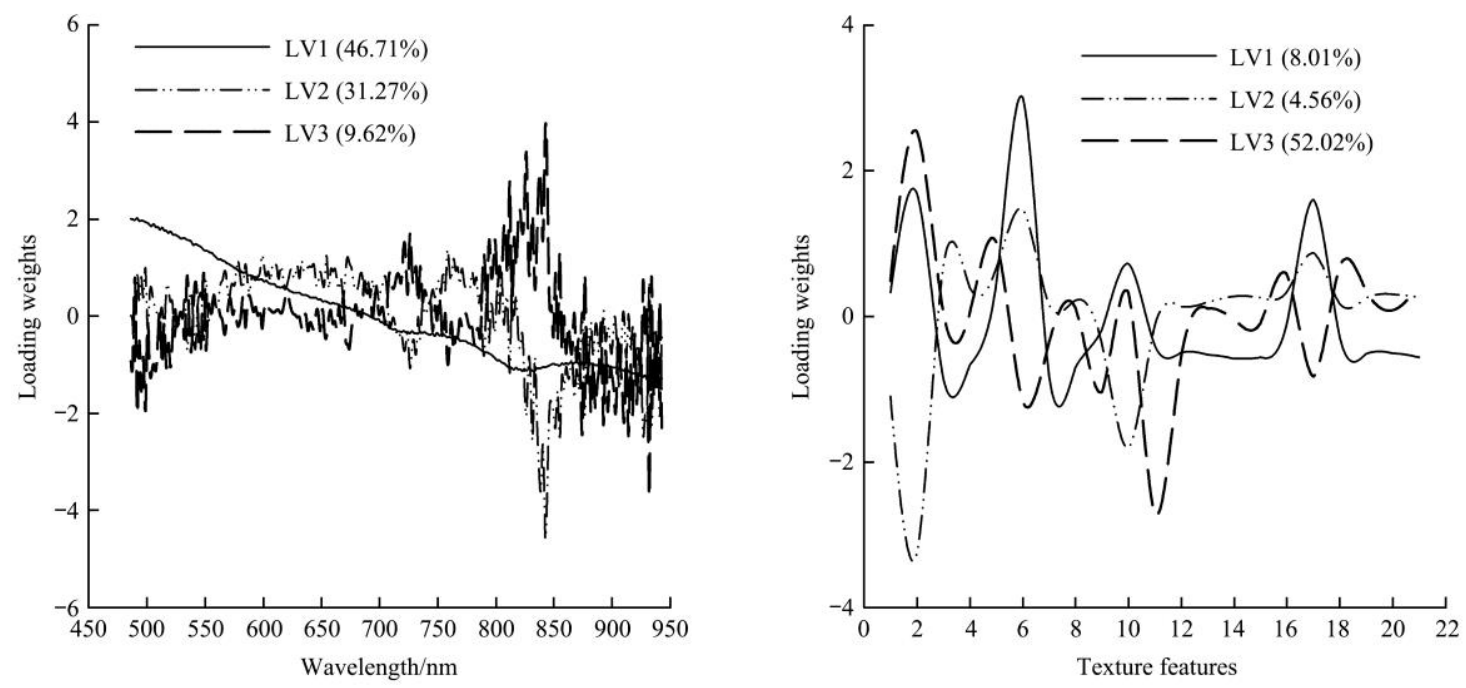

a. Spectral information

b. Textural information

Figure 7 Loading weights of the first three LVs of spectral information and textural information

According to Figure $7 \mathrm{a}$, the loading weights ofLV1 generally produced a decreasing trend in wavelength that might have been caused by the increasing trend of reflectance deviation shown in Figure 4. Significant peaks can be seen in the loading weights of LV2 and LV3 around $840 \mathrm{~nm}$. That was considered an important feature to establish the equation for the soil line ${ }^{[29]}$ and has a strong correlation with soil properties, such as $\mathrm{SMC}^{[30]}$. A strong negative band was observed near $940 \mathrm{~nm}$ of LV3, which was probably due to the $\mathrm{OH}$ bending for the water molecules ${ }^{[31,32]}$.

Figure $7 \mathrm{~b}$ showed the loading weights for the first three LVs of the 21 textural features. Significant peaks were observed for features $2,6,10,11$ and 17 , which appear in bold font in Table 3. It can be inferred that the entropy of PC1 and PC2 makes a large contribution to predicting SMC. This might because the characteristic of soil surface particles is largely affected by SMC. The $\sigma$ of energy and peak of PC1 also makes a moderate contribution to predicting SMC.

\section{Conclusions}

(1) Thirty-six sandy soil samples with variable SMC levels were produced with progressive natural evaporation. An HSI system was developed to collect spectral $(400-1000 \mathrm{~nm})$ and spatial $(1620 \times 841$ pixels $)$ 
data of soil samples.

(2) Overall, 256 optimal spectral wavelengths obtained using the averaged reflectance of ROI with high SNR values were selected and smoothed with the S-G filter. Twenty-eight textural features were extracted using a GLCM, and 21 of them were used to predict SMC, with the absolute value of coefficient surpassing 0.5.

(3) Six LVs extracted from the spectral information, 4 LVs extracted from the textural information and fused data were used to build regression models with a three-layer BPNN. The $R_{C}^{2}$ was $0.9532,0.9386$ and 0.9492 for the three calibration models, and the $R_{P}^{2}$ was $0.9126,0.7196$ and 0.9350 for the validations.

The results of the present study indicate that applying a combination of spectral and textural information from a hyperspectral image can improve the ability to predict SMC. The results also suggest that reducing data redundancy and collinearity is essential to making such predictions more accurate. This will be the focus of future research. In addition, for future experiments, the number of soil samples will be increased to make the regression model more stable and robust. Measurements made during field conditions will also be investigated further.

\section{Acknowledgement}

This research was financially supported by International Science and Technology Cooperation Project of China Ministry of Agriculture (2015-Z44).

\section{[References]}

[1] Whiting M L, Li L, Ustin S L. Predicting water content using Gaussian model on soil spectra. Remote Sensing of Environment, 2004; 89(4): 535-552.

[2] Lesaignoux A, Fabre S, Briottet X. Influence of soil moisture content on spectral reflectance of bare soils in the 0.4-14 $\mu \mathrm{m}$ domain. International Journal of Remote Sensing, 2013; 34(7): 2268-2285.

[3] Jiang P A. Measuring soil water content by using near infrared spectral characteristics of soil. Spectroscopy and Spectral Analysis, 2012; 32(8): 2117-2121. (in Chinese)

[4] Wu D, Fan W, Cui Y, Yan B, Xu X. Review of monitoring soil water content using hyperspectral remote sensing. Spectroscopy and Spectral Analysis, 2010; 30(11):
3067-3071. (in Chinese)

[5] Yao Y, Wei N, Tang P, Li Z, Yu Q, Xu X, et al. Hyper-spectral characteristics and modeling of black soil moisture content. Transactions of the CSAE, 2011; 27(8): 95-100. (in Chinese)

[6] Peng J, Shen H, He S W, Wu J S. Soil moisture retrieving using hyperspectral data with the application of wavelet analysis. Environmental Earth Sciences, 2013; 69(1): 279-288.

[7] Oltra-Carrió R, Baup F, Fabre S, Fieuzal R, Briottet X. Improvement of soil moisture retrieval from hyperspectral VNIR-SWIR data using clay content information: From laboratory to field experiments. Remote Sensing, 2015; 7(3): 3184-3205.

[8] Park B, Lu R. Hyperspectral imaging technology in food and agriculture. Springer New York, 2015.

[9] Rourke S M O, Holden N M. Optical sensing and chemometric analysis of soil organic carbon - a cost effective alternative to conventional laboratory methods? Soil Use and Management, 2011; 27(2): 143-155.

[10] Zhang H. Soil nutrition content and type measurement based on NIR spectrum and hyper spectra image technology and design portable instrument. Hangzhou: Zhejiang University, 2015. (in Chinese)

[11] O'Rourke S M, Holden N M. Determination of soil organic matter and carbon fractions in forest top soils using spectral data acquired from visible-near infrared hyperspectral images. Soil Science Society of America Journal, 2012; 76(2): 586-596.

[12] Burud I, Moni C, Flo A, Futsaether C, Steffens M, Rasse D P. Qualitative and quantitative mapping of biochar in a soil profile using hyperspectral imaging. Soil and Tillage Research, 2016; 155: 523-531.

[13] Pessoa L G M, Dos Santos Freire M B G, Wilcox B P, Rossi C G, Souza A M D O, Galvíncio J D. Spectral reflectance characteristics of laboratory-grown salt crusts on silty clay and sandy soils. Communications in Soil Science and Plant Analysis, 2015; 46(15): 1895-1904.

[14] Gomez C, Drost A P A, Roger J M. Analysis of the uncertainties affecting predictions of clay contents from VNIR/SWIR hyperspectral data. Remote Sensing of Environment, 2015; 156: 58-70.

[15] Li S, Wang S, Shi Z. Prediction of vertical distribution of soil nitrogen content in soil profile using spectral imaging technique. Acta Pedologica Sinica, 2015; 52(5): 1014-1023. (in Chinese)

[16] GB/T. Method for determination of soil water content. PRC National Standard, 1987. (in Chinese)

[17] Dong G, Guo J, Wang C, Chen Z L, Zheng L, Zhu D Z. The classification of wheat varieties based on near infrared 
hyperspectral imaging and information fusion.

Spectroscopy and Spectral Analysis, 2015; 35(12):

3369-3374. (in Chinese)

[18] Steffens M, Buddenbaum H. Laboratory imaging spectroscopy of a stagnic Luvisol profile-High resolution soil characterisation, classification and mapping of elemental concentrations. Geoderma, 2013; 195: 122-132.

[19] Cen H, Lu R, Zhu Q, Mendoza F. Nondestructive detection of chilling injury in cucumber fruit using hyperspectral imaging with feature selection and supervised classification. Postharvest Biology and Technology, 2016; 111: 352-361.

[20] Rourke S M O, Holden N H. Determination of soil organic matter and carbon fractions in forest top soils using spectral data acquired from visible-near infrared hyperspectral images. Soil Science Society of America Journal, 2012; 76: 586.

[21] Savitzky A, Golay M J E. Smoothing and differentiation of data by simplified least squares procedures. Analytical Chemistry, 1964; 36: 1627-1639.

[22] Li H W, Li H, He Jin, Li S W. Measuring system for residue cover rate in field based on BP neural network. Transactions of the CSAM, 2009; 40(6): 58-62. (in Chinese)

[23] Janik L, Cozzolino D, Dambergs R, Cynkar W, Gishen M. The prediction of total anthocyanin concentration in red-grape homogenates using visible-near-infrared spectroscopy and artificial neural networks. Analytica Chimica Acta, 2007; 594(1): 107-118.

[24] Ji W J, Li X, Li C X, Zhou Y, Shi Z. Using different data mining algorithms to predict soil organic matter based on visible-near infrared spectroscopy. Spectroscopy Spectral
Analysis, 2012; 32: 2393-2398. (in Chinese)

[25] Mouazen A M, Kuang B, de Baerdemaeker J, Ramon H. Comparison among principal component, partial least squares and back propagation neural network analyses for accuracy of measurement of selected soil properties with visible and near infrared spectroscopy. Geoderma, 2010; 158: 23-31.

[26] Widyanto M R, Nobuhara H, Kawamoto K, Hirota K, Kusumoputro B. Improving recognition and generalization capability of back-propagation NN using a self-organized network inspired by immune algorithm (SONIA). Applied Soft Computing Journal, 2005; 6: 72-84.

[27] Janik L J, Merry R H, Skjemstad J. Can mid infrared diffuse reflectance analysis replace soil extractions? Animal Production Science, 1998; 38(7): 681-696.

[28] Ji W J, Adamchuk V I, Biswas A, Dhawale N M, Sudarsan B, Zhang Y K, et al. Assessment of soil properties in situ using a prototype portable MIR spectrometer in two agricultural fields. Biosystems Engineering, 2016; 152: 14-27.

[29] Baret F, Jacquemoud S, Hanocq J. The soil line concept in remote sensing. Remote Sensing Reviews, 1993; 7(1): 65-82.

[30] Peng J, Xiang H Y, Zhou Q, Zhang Y Z. Influence of soil iron oxide on VNIR diffuse reflectance spectroscopy. Spectroscopy and Spectral Analysis, 2013; 33(2): 502-506. (in Chinese)

[31] Stenberg B, Rossel R A V, Mouazen A M, Wetterlind J. Chapter five-visible and near infrared spectroscopy in soil science. Advances in agronomy, 2010; 107: 163-215.

[32] Galvão L S, Vitorello Í. Variability of laboratory measured soil lines of soils from southeastern Brazil. Remote Sensing of Environment, 1998; 63(2): 166-181. 